\title{
Salomon Christine, Savoirs et pouvoirs thérapeutiques kanaks
}

2000, Paris, PUF, INSERM (« Ethnologies »), 159 p.

Élisabeth Motte-Florac

\section{(2) OpenEdition}

\section{Journals}

Édition électronique

URL : http://journals.openedition.org/jso/1627

DOI : $10.4000 /$ jso. 1627

ISSN : $1760-7256$

Éditeur

Société des océanistes

Édition imprimée

Date de publication : 1 décembre 2001

Pagination : 221-222

ISSN : 0300-953x

Référence électronique

Élisabeth Motte-Florac, "Salomon Christine, Savoirs et pouvoirs thérapeutiques kanaks », Journal de la Société des Océanistes [En ligne], 113 | Année 2001-2, mis en ligne le 27 mai 2008, consulté le 24 septembre 2020. URL : http://journals.openedition.org/jso/1627 ; DOI : https://doi.org/10.4000/jso. 1627 


\section{COMPTES RENDUS}

Salomon Christine, 2000. Savoirs et pouvoirs thérapeutiques kanaks. Paris, PUF, INSERM ( Ethnologies »), $159 \mathrm{p}$.

Toute thérapeutique est, par définition, profondément complexe dans la mesure où elle concerne l'être humain dans sa complétude. Pluridimensionnelle, elle réclame du chercheur qu'il l'aborde dans les différents registres qui la constituent et qu'il explore les différents éléments, représentations, concepts qui la fondent. Mais elle exige aussi, après un tel découpage, que l'on reconstitue l'architecture qui sous-tend l'ensemble conceptuel et que l'on prenne en considération sa constante évolution. L'ampleur d'une telle tâche impose des choix qui conduisent inévitablement à des simplifications, des appauvrissements; quel que soit l'éclairage adopté pour étudier la thérapeutique et pour aussi intéressant qu'il soit, il crée inévitablement des zones d'ombre. Le travail de Christine Salomon est, comme celui des chercheurs qui l'ont précédée et dont elle fait mention, représentatif d'une époque, d'une formation, d'une personnalité. Se fondant sur les textes de tradition orale qu'elle a recueillis et s'appuyant sur des considérations de différents ordres -_ dont linguistique (en ajië comme en paicî, les idées de vie, de guérison et de descendance sont exprimées par un seul et même terme) - elle privilégie les notions d'ancêtres, d'appartenance, d'identité, et les considère comme d'incontournables repères à partir desquels chacun des actes de la vie et de la thérapeutique font sens. Partant, C. Salomon engage son étude en mettant l'accent sur l'importance de deux cadres d'appartenance (clan et lignage d'une part, chefferie d'autre part) et sur l'omniprésence à la fois des forces ancestrales agnatiques et de l'ascendance utérine dans l'espace de vie et les temps de maladie. Elle nous offre une étude originale et rigoureuse autour de ces fondements sociaux et culturels des pratiques thérapeutiques traditionnelles kanak. Dans un souci constant de permettre au lecteur d'aborder de façon pertinente les représentations locales, de nombreux extraits d'entretiens sont retranscrits et intelligemment mis en perspective par les données linguistiques nécessaires à la compréhension de la formulation des pensées.

L'ouvrage, agréablement présenté, est organisé en trois parties composées, chacune, de deux chapitres. La première partie est consacrée aux représentations kanak de la filiation, de l'hérédité, du corps et de ses substances, sans lesquelles il est impossible de cerner la notion de personne. Dans ces langues, « le discours sur la médecine renvoie nécessairement à la question de la vie et à celle de ses origines tant à une échelle qui procède d'une vision phylogénétique qu'à l'échelle ontogénétique, celle de l'individu et des constituants de la personne humaine. » (p. 19) L'auteur insiste sur l'importance des êtres des origines (responsables du début de la civilisation par le processus complexe d'hominisation, et de la survie des êtres humains) et des ancêtres (qui font naître et grandir un individu, et prennent soin de lui). Les pouvoirs de ces derniers, matérialisés dans des pierres et plantes magiques - le trésor du clan —, peuvent être utilisés pour nourrir, protéger, soigner, mais aussi pour anéantir. L'enfant, formé à partir du sang de sa mère et de celui de son père, sera protégé — de sa conception à sa mort — par les forces ancestrales du clan, «totems » et « esprits des morts ». Leur bienveillance lui permettra de s'accomplir physiquement, mentalement et socialement, à moins qu'il ne déroge gravement à l'éthique du groupe. On l'aura compris, l'auteur nous présente toutes les données qui permettent d'envisager « l'omnipotence du monde ancestral » comme une clef indispensable pour appréhender les notions de maladies, et de malheurs individuels et sociaux; omnipotence qui " amène chaque être humain à se penser dans une filiation qui le relie à ses ancêtres auxquels il est redevable de la vie et de tout ce qui est nécessaire à son entretien » (p. 33).

Une deuxième partie fait le point sur les représentations de la maladie. On retrouve chez les Kanaks, comme ailleurs, la notion fondamentale d'interdépendance entre ancêtres et vivants, nature, société et individu, externe et interne, soma et psyché, etc. En raison de ces liens multiples, toute rupture d'équilibre désorganise non seulement le rapport de la personne à elle-même mais aussi celui de la personne à son environnement naturel et social. Mais chez les Kanaks, au-delà de la personne malade et de son entourage immédiat (conjoint, enfants), c'est le lignage - voire le clan et ses alliés — qui est atteint et déséquilibré par l'événement morbide. On conçoit dès lors que la parenté au sens large (parents et alliés) se mobilise pour trouver les thérapeutes adéquats mais aussi pour rendre visite au malade et lui prodiguer des encouragements par des paroles et des présents.

C. Salomon aborde ensuite les différents types de maladies en prenant en compte, de façon avisée, fondements culturels, théories locales explicatives du malheur, données linguistiques, etc. Maladies indigènes, nouvelles, vraies, graves, contagieuses, provoquées par 
les ancêtres, fabriquées..., constituent des catégories non hermétiques qui peuvent se recouper en fonction des interprétations données à l'apparition et à l'évolution de la maladie. Une grande constante, plus particulière à la société kanak, est la notion de conflit qui est manifeste dans la plupart des syndromes. Le vocabulaire de la violence guerrière domine toute la terminologie médicale et la rivalité, omniprésente, ne concerne pas seulement les rapports entre des entités groupales (lutte armée ou magique) mais s'applique aussi aux relations interpersonnelles qui sont dominées par « la jalousie».

Les informations permettant de diagnostiquer une maladie relèvent essentiellement de son étiologie, ce qui implique que le choix du traitement est établi de façon relativement indépendante de la nosologie. Les causes des maladies sont d'autant plus complexes qu'un même phénomène peut être attribué à des causes différentes selon le contexte et une même causalité est susceptible de provoquer des effets divers : maladie, conflit social, accident climatique, etc. Pluralité des causes et des effets, agglutination des maladies qui sont superposées ou surajoutées les unes aux autres, offrent une certaine liberté pour interpréter chaque cas.

Dans la dernière partie, C. Salomon aborde les moyens par lesquels la société kanak diagnostique et soigne les maux qui affectent ses membres et tente d'analyser ce qui est en jeu dans les modèles thérapeutiques proposés. Un premier chapitre (chap. 5) « Voir la maladie » présente les thérapeutes locaux et leurs modes de connaissance — initiatique et empirique ; les voyants et devins «voient la maladie », les thérapeutes «manuels" «touchent pour voir». Comme dans nombre d'autres sociétés, la capacité de « voir » la maladie peut être « incorporée à son détenteur » en faisant intervenir révélation ou rêve, ou être transmise par initiation ; elle est utilisée pour des motifs extrêmement divers (maladies, disparitions, entreprises passées ou futures...). Mais chez les Kanaks, cette capacité (pouvoirs et savoirs) est spécifiquement masculine et lignagère, et en étroite relation avec l'ordre politique. La catégorie des thérapeutes qui « touchent pour voir » est, quant à elle, ouverte aux hommes comme aux femmes; le savoir est transmis, lui aussi, au sein du lignage mais peut s'enrichir d'apports exogènes et divers. Lors du diagnostic, le rôle de ces thérapeutes n'est pas de dire si le malade va guérir ou si l'affaire va réussir mais de savoir quel lignage, quel individu est en cause et de suggérer « ce qu'il convient de faire pour être en conformité avec les règles sociales et, par conséquent, pour se donner les meilleures chances de réussir. » (p. 111)

Un deuxième chapitre (chap. 6) « Défaire la maladie » expose les procédés utilisés dans le traitement des maladies, leurs particularités temporelles et les interdits qui leur sont associés. Mais quelle que soit la pratique, « l'invocation des ancêtres prime sur la médication. » La parole constitue le support de la communication avec le monde ancestral mais d'autres actes (bénir, « souffler », etc.) sont à considérer comme des modulations de cette communication orale. Parmi les médications, les plantes médicinales constituent un savoir du groupe lignager auquel tout le monde a accès mais si la femme peut « emporter » avec elle les plantes patrimoniales après son mariage, elle ne pourra ni les transmettre à ses enfants, ni s'en servir hors du groupe (cet exercice est une spécialisation masculine). Seul le pouvoir ancestral transmis par le thérapeute — «la puissance de sa parole »- confère aux plantes leur efficience (bénéfique ou maléfique).

L'auteur conclut son ouvrage en évoquant les problèmes posés par l'importation de nouveaux concepts à travers la colonisation, l'arrivée des missionnaires, l'apparition de la biomédecine, et les formes d'adaptation des Kanaks à ces nouvelles conditions : assouplissement des règles de conduite, mouvement vers l'individualisation, etc.

Cet intéressant ouvrage, en privilégiant avec justesse l'importance des références généalogiques, des appartenances et des conflits, offre une analyse originale de la thérapeutique traditionnelle kanak. Souhaitons qu'après cette « première recherche » comme elle le dit elle-même, C. Salomon s'engage plus avant dans l'observation et l'analyse des savoirs et pouvoirs thérapeutiques kanak.

\section{Élisabeth MOTTE-FLORAC}

Université Montpellier 1, CNRS-LACITO, Villejuif

Salomon Christine, 2000. Savoirs et pouvoirs thérapeutiques kanaks. Paris, PUF, INSERM («Ethnologies »), $159 \mathrm{p}$.

«Comment, en Nouvelle-Calédonie, les gens de la Grande-Terre - du moins ceux de la région centrenord, aux marges des aires a'jië et paicî — perçoiventils les faits de maladie, quelles explications en donnentils et quels modes de résolution de ces faits ont-ils élaborés?»

À ces questions posées en introduction, le livre de Christine Salomon apporte des réponses détaillées et argumentées que tous ceux qui travaillent en Nouvelle-Calédonie, ethnologues comme soignants, ont déjà pu depuis plusieurs années apprécier à leur juste valeur. En effet, le manuscrit, sous la forme d'un rapport d'enquête daté de 1993, a beaucoup circulé dans le pays, suscitant l'intérêt bien au-delà du petit milieu des chercheurs. Sur le sujet, on ne disposait jusque-là, comme l'indique l'auteur lui-même, que de notations éparses chez les premiers missionnaires et voyageurs, de trois chapitres des Notes d'ethnologie de Leenhardt (1933) sur les « Totems », les « Dieux » et la " Magie ", de la thèse pour le moins affligeante d'Éliane Métais sur La sorcellerie canaque actuelle (1967) en pays tirî et de quelques textes de l'ethnobotaniste Dominique Bourret sur la pharmacopée traditionnelle. Le rapport de Christine Salomon nous livra une monographie d'une rare qualité, à la fois novatrice, systématique et documentée, dans laquelle la précision de la description ethnographique n'a d'égale que la prudente (parfois même trop prudente) rigueur des analyses proposées. Il faut se réjouir aujourd'hui de sa publication chez un éditeur prestigieux qui permettra de faire plus largement connaître cette contri- 